\title{
Challenges in progressing schizophrenia therapy
}

\section{Mihály Hajós*}

Department of Neuroscience, Pfizer Global Research and Development, Groton, CT, USA

*Correspondence: mihaly.hajos@pfizer.com

Some of the most famous and beloved artworks were created by artists who suffered from serious mental disorders, such as schizophrenia. One of my favorite paintings is The Solitary Cedar by the Hungarian artist Tivadar Kosztka Csontváry (Figure 1). Csontváry lived at the turn of the 19th century under the Austro-Hungarian Empire, and worked as a licensed pharmacist until he heard voices insisting that he give up his civil profession and devote his life to painting. He traveled around Europe, studied painting and developed a very individual style. Some of his most amazing works were created in a relatively short period of time. I have been wondering since my student years, how much his illness, his schizophrenia contributed to his work. This question could be debated either from an aesthetic or a scientific point of view. Was his schizophrenia fundamental to his creativity; did his illness define his individual artistic style? If yes, then one can argue that treatment of his condition could have fundamentally changed the way he painted. On the other hand, schizophrenia also prevented him reaching his full potential as an artist. We know that his creative period was shortlived as his illness progressed rapidly and he was not able to complete his paintings. Eventually he could not paint at all.

Would Csontváry have been luckier living in our recent time? Would currently available therapies have helped him finish his paintings? Difficult to answer. No one questions the progress psychiatry has made over the last century. Diagnostic accuracy has improved with clinically better defined sub-types of the disease. The discovery, more than 50 years ago, that the antihistamine agent, chlorpromazine calmed agitated psychiatric patients opened a new era in psychiatry and antipsychotic drug research. It was soon recognized that chlorpromazine and other related compounds (referred to as neuroleptics) induced not only sedation but also a diminution of positive symptoms of schizophrenia, such as hallucinations and delusions. The subsequent recognition of a strong correlation between clinical potency of neuroleptic agents and their ability to block dopamine D2-type receptors suggests that dopaminergic hyperactivity might represent a critical pathophysiological event in schizophrenia. Consistent with this, dopaminergic hyperactivity has been confirmed in schizophrenia patients using in vivo imaging studies, and all current agents for treating schizophrenia function primarily by blocking dopamine D2 receptors.

However, dopaminergic hyperactivity is seen only in a subset of patients during the acute stages of the illness, and it is now generally understood that dopamine is not the only (or even the main) dysfunctional neurotransmitter system in this disease. In fact, in addition to dopamine, practically all known classical neurotransmitters, or at least their receptors have been linked to the disease, including glutamate, GABA, serotonin, norepinephrine, acetylcholine and a variety of neuropeptides and endogenous neuromodulators, such as the endorphins and endocannabinoids. Genetic studies have so far contributed little for clarifying and understanding the critical neurotransmission abnormalities in the disease, although several affected genes (including neuregulin, dysbindin, DISC1, DAAO, GRM3, and G72) may indicate a dysfunctional NMDA receptor-mediated glutamate transmission. Considering the plasticity and dynamic feature of the brain, one can argue that alteration in any of the main brain neurotransmitter systems would subsequently influence other neurotransmitters as well. Therefore, probably the best approach to understand brain dysfunctions associated with schizophrenia would be based on neuronal circuits, where interactions between various neurotransmitter systems identified within specific neuronal pathways and networks could be integrated.

It is recognized that schizophrenia is a genetically predisposed disease, and like other complex disorders it is characterized by the contribution of multiple risk genes in combination with epigenetic and environmental processes leading to the development of the disease. However, it is still a major challenge for the forthcoming years to determine whether a relatively few highly penetrant but rare major genetic deficits would determine the disease, or a large number of genes would contribute although each gene accounting for only a small increment in risk. Interestingly, a recent genome-wide search of copy number variants (CNVs) revealed association between CNVs of certain high penetrant genes not only with schizophrenia, but also other psychiatric illnesses, such as autism and bipolar disorder. These findings might point to the fact that most psychiatric diseases share a number of symptoms therefore these genetic abnormalities might correlate better with symptoms of various psychiatric disorders rather than with a singular psychiatric disease itself. The concept and application of endophenotypes better define this relationship: endophenotypes capture and measure pathophysiological signals contributing to the clinical manifestation of the disease. Neurophysiological abnormalities associated with schizophrenia could be considered as endophenotypes; their value in genetic studies of schizophrenia has already been demonstrated. Future research to model neurophysiological abnormalities of schizophrenia (pathophysiological endophenotypes) in animal experiments will be required to use these endophenotypes as translational biomarkers to bridge experimental studies to clinical development.

During the last two or three decades, huge investments have been made in schizophrenia research by academic institutes as well as the drug industry, with only modest progress. In clinical practice a wide array of safe antipsychotics exist with differentiated side-effect profiles. However, many critical symptoms of the disease are still not impacted by the current treatments. Most significantly, it has been recognized that patients' cognitive function is the main factor determining their everyday functioning in the society. No drugs currently address this critically important deficiency 


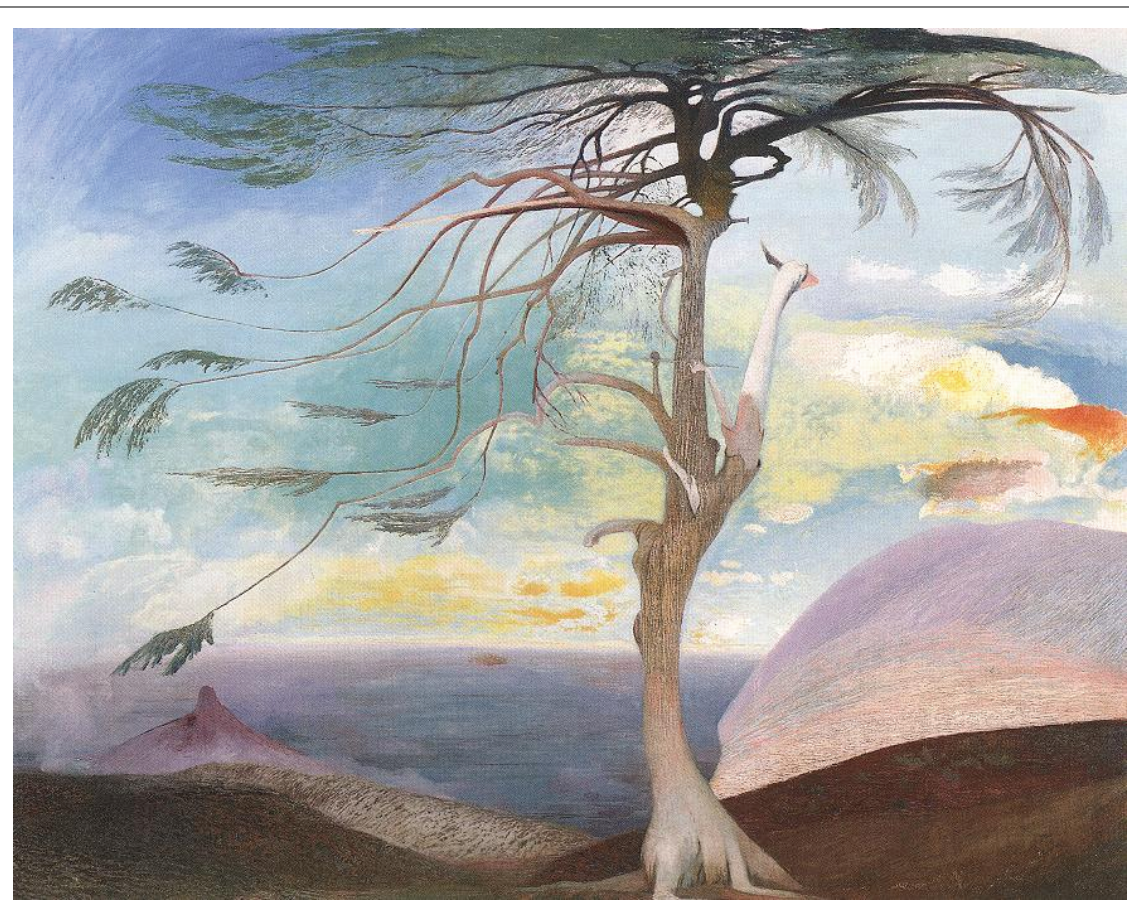

FIGURE 1 | Csontváry:The Solitary Cedar (Csontváry Museum, Pécs, Hungary).

in schizophrenia, and most of the clinical trials fail in this field. Are we after the right therapeutic targets? Do we undermine our efforts by not defining schizophrenia better, recognizing genetically based sub-types of the disease? While genetically-based personalized therapy is an attractive concept, still very little is happening in practice e.g. designing the best pharmacotherapy for patients based on their individual genetic characteristics.

Hypothesis-driven research is the cornerstone of scientific discovery, but sometimes a hypothesis can become a dogma, slowing down the progress of discovery. Sometimes our experimental findings do not mold easily into our pre-fabricated concepts and hypothesis. I hope that Frontiers in Schizophrenia will support all the efforts which question current status quo and it will provide a forum for new ideas and open debate. We need new approaches and new concepts. We need them for our patients so that they can pursue whatever they want to do in their life, if not for any other reason than just to paint lonely cedars in Lebanon.

Received: 02 March 2010; accepted: 02 March 2010; published online: 17 March 2010.

Citation: Hajós M (2010) Challenges in progressing schizophrenia therapy. Front. Psychiatry 1:9. doi: 10.3389/ fpsyt.2010.00009

This article was submitted to Frontiers in Schizophrenia a specialty of Frontiers in Psychiatry.

Copyright $(\odot) 2010$ Hajós. This is an open-access article subject to an exclusive license agreement between the authors and the Frontiers Research Foundation, which permits unrestricted use, distribution, and reproduction in any medium, provided the original authors and source are credited. 